\title{
Operating modes of dual-grating dielectric laser accelerators
}

\author{
Dylan S. Black $\odot,{ }^{1, *}$ Zhexin Zhao, ${ }^{1, *}$ Kenneth J. Leedle $\odot,{ }^{1}$ Yu Miao, ${ }^{1}$ \\ Robert L. Byer®, ${ }^{2}$ Shanhui Fan, ${ }^{1}$ and Olav Solgaard $\odot^{1}$ \\ ${ }^{1}$ Department of Electrical Engineering, Stanford University, \\ 350 Serra Mall, Stanford, California 94305-9505, USA \\ ${ }^{2}$ Department of Applied Physics, Stanford University, 348 Via Pueblo Mall, Stanford, \\ California 94305-4090, USA
}

(Received 14 April 2020; accepted 21 October 2020; published 10 November 2020)

\begin{abstract}
In this work, we present a comprehensive theoretical description of the Lorentz force in dual-grating dielectric laser accelerators (DLA). Here we examine the dual-grating DLA under arbitrary illumination conditions, both single-side and dual-side drive. We improve upon previous descriptions of these forces by providing a unified description of all possible operating modes of these devices, and classify the modes into three categories. In particular, we predict the existence of the previously observed "line modes" and a novel "elliptical mode", provide an experimental demonstration of this new mode, and suggest a possible application for it in improving the resolution of attosecond-scale streak cameras. Finally, we examine several complementary methods of tuning a dual-grating DLA to a desired operating mode-particularly dual-drive phase rotation and translational offset of the grating teeth.
\end{abstract}

DOI: 10.1103/PhysRevAccelBeams.23.114001

\section{INTRODUCTION}

The dielectric laser accelerator (DLA) is an opticalfrequency linear accelerator that uses the high electric fields of pulsed lasers to increase acceleration gradients over conventional radio-frequency (rf) linacs by more than an order of magnitude [1]. Recently, dual-grating, dual-drive DLAs [2] have emerged as a useful and flexible DLA architecture. Through tuning of the particle injection phase and the dual-drive illumination phase, they can provide acceleration [2,3], transverse focusing [4], microbunching [5], and transverse deflection [2,5].

The fields and forces in these DLA structures have been studied previously, and the resulting equations used to design electron beam control and confinement schemes [4-9]. Previous literature has examined the single-sided plane wave illumination of dual-grating structures in great detail, as well as mirror-symmetric dual-sided plane wave illumination [5,10-16]. In dual-drive structures, symmetric illumination of a symmetric dual grating produces a symmetric Lorentz force distribution providing primarily longitudinal momentum modulation. Symmetric illumination produces a "cosh mode", which gets its name from the hyperbolic cosine shape of the longitudinal force.

*D. S. B. and Z. Z. contributed equally to this work.

Published by the American Physical Society under the terms of the Creative Commons Attribution 4.0 International license. Further distribution of this work must maintain attribution to the author(s) and the published article's title, journal citation, and DOI.
Conversely, antisymmetric illumination of the symmetric grating produces a transversely deflecting "sinh mode". The cosh and sinh modes are referred to as the principal modes of dual grating operation.

Recently, DLA operating modes providing coupled longitudinal and transverse momentum modulation ("skew modes") have been experimentally demonstrated $[2,4]$. Because these modes are less obviously useful for an accelerating beamline, they have not received nearly as much attention (either theoretically or experimentally) as the more commonly seen principal DLA modes. However, current theoretical frameworks derived for the principal operating modes do not adequately describe the phase space profile of skew modes. In particular, the theory presented in $[2,4,11]$ and others do not correctly predict the linelike character of the skew modes experimentally demonstrated thus far, instead predicting the existence of an elliptical mode.

It is therefore of great importance to comprehensively investigate the Lorentz force in dual-grating DLAs under general illumination conditions (particularly general dualdrive conditions), and establish a framework for mapping the characteristics of a dual-grating DLA onto its operational mode, and thus its effect on an electron beam.

In this work, we present a general analytic description of the electromagnetic fields and forces on a particle in a dualgrating DLA under single-side and dual-side plane wave illumination. We derive the fields and forces present in the most common devices, symmetric dual gratings, discuss the symmetries of the resulting equations, then generalize them to encompass nonmirror-symmetric dual gratings and 
arbitrary illumination conditions. We give a unified description of both the principal modes and skew modes, and find that three classes of skew modes exist: the linelike mode which has been demonstrated in previous experiments and two elliptical modes which have never been observed-an upright ellipse and a rotated ellipse.

Guided by the unified description, we discuss various methods of tuning between these operational modes, including dual-drive relative phase and the "glide ratio" where the grating teeth of one row are offset relative to the other. We further employ symmetry arguments to show the correspondence between tuning methods in the dual-drive and single-side drive cases. We also present experimental confirmation of the previously unobserved elliptical skew mode in a single-side drive configuration. The practical importance of this mode is due to its potential to improve the resolution of attosecond-scale streak cameras.

\section{DERIVATION OF DUAL DRIVE FIELDS}

In this section, we derive the Lorentz force seen by a phase-matched electron inside a dual-grating accelerator structure. Although part of the derivation has been previously presented by Black et al. [5], we present it again here as a foundation for the more complex analysis of the symmetries of the force equations and the phase space of DLA operation modes.

We consider a dual-grating structure as shown in Fig. 1. We define a right-handed coordinate system with $z$ along the axis of electron beam propagation and $y$ the transverse coordinate. The structure is assumed to be quasi-infinite in the $x$ direction, allowing the problem to be treated in 2D. The 2D approximation captures the transverse shape of the modes [2], though 3D effects should be taken into account to accurately calculate the coupling strength of the incident lasers into the structure modes and the $x$-dependence of the field.

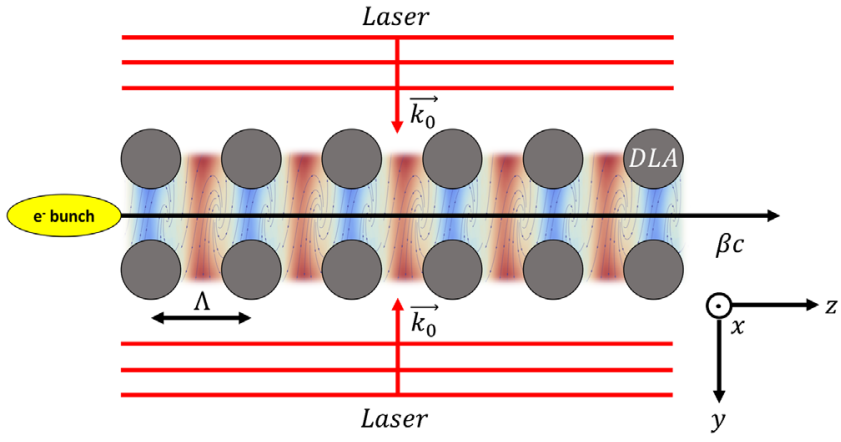

FIG. 1. An electron bunch travels along $z$ through a dualgrating DLA structure. The grating has periodicity $\Lambda$ and is assumed infinite in $x$. Two lasers of equal amplitude with relative phase $\theta_{r}$ are normally incident on the grating structure, with wave vectors $\pm k_{0} \hat{y}$. A reference particle enters the structure traveling with speed $\beta c$ at some injection phase $\alpha$ relative to the laser field, and experiences a force given by Eq. (20).
In 2D problems, the electric and magnetic fields can be separated into orthogonal TE and TM modes, where the TM mode can be fully described by $H_{x}$, and the TE mode by $E_{x}$. We assume harmonic time dependence of the electric and magnetic fields, writing them as $\mathbf{E}(\mathbf{r}, t)=\mathbf{E}(\mathbf{r}) e^{-i \omega t}$, $\mathbf{H}(\mathbf{r}, t)=\mathbf{H}(\mathbf{r}) e^{-i \omega t}$. We assume an isotropic, linear dielectric medium with permeability $\mu=\mu_{0}$ and permittivity $\epsilon=\epsilon(\mathbf{r})$.

The $\mathbf{H}$ field must obey the wave equation [17]:

$$
\nabla \times\left(\frac{1}{\epsilon(\mathbf{r})} \nabla \times \mathbf{H}\right)=\mu_{0} \omega^{2} \mathbf{H}
$$

The dual-grating structure is periodic in $z$ with periodicity $\Lambda$. Therefore, the solutions to Eq. (1) must satisfy the Floquet-Bloch theorem

$$
\begin{aligned}
\mathbf{H}(\mathbf{r}) & =\mathbf{H}_{p}(\mathbf{r}) e^{i k_{z} z}, \\
\mathbf{H}_{p}(\mathbf{r}+\Lambda \hat{\mathbf{z}}) & =\mathbf{H}_{p}(\mathbf{r}),
\end{aligned}
$$

where $k_{z}$ is a Bloch wave vector.

Being periodic, $\mathbf{H}_{p}(\mathbf{r})$ may be expanded in a Fourier series

$$
\mathbf{H}_{p}(\mathbf{r})=\sum_{n=-\infty}^{\infty} \mathbf{h}_{n}(x, y) e^{i k_{n} z}
$$

where $k_{n}=2 \pi n / \Lambda$. Note that we have defined two different wave vectors: $k_{n}$, the propagation constant associated with the $z$ variation of the field's $n$th spatial harmonic, and $k_{z}$, the Bloch wave vector associated with the phase advance of the entire wave through the periodic DLA structure.

In this section we consider in detail the fields and forces in the TM case. The TE modes are treated similarly and are detailed in the Supplemental Material [18].

\section{A. Single side illumination}

The TM modes can be described by $H_{x}$, and the $x$-invariance of the problem restricts the coordinate dependence of $\mathbf{h}_{n}(x, y)$ to $\mathbf{h}_{n}(y)$. Thus, in the TM mode, the magnetic field can be completely described by a single scalar function of the form

$$
H_{x}=\sum_{n} H_{x}^{(n)}=\sum_{n=-\infty}^{\infty} h_{n}(y) e^{i\left(k_{n}+k_{z}\right) z},
$$

where $\mathbf{H}(\mathbf{r})=H_{x} \hat{\mathbf{x}}$.

The dual-grating structure is illuminated by a $z$-polarized plane wave propagating in the $+y$ direction with electric field amplitude of $E_{0}$. Its $\mathbf{H}$ field is 


$$
\mathbf{H}_{0}(\mathbf{r}, t)=\frac{E_{0}}{\mu_{0} c} e^{i\left(k_{0} y-\omega t\right)} \hat{\mathbf{x}},
$$

where $k_{0}=\omega / c$ is the vacuum wave vector.

The plane wave scatters off the dual-grating structure, producing fields in the channel gap whose $n$th spatial harmonic is

$$
H_{x}^{(n)}=\frac{E_{0}}{\mu_{0} c} \frac{k_{0}}{\Gamma_{n}}\left(d_{n} e^{-\Gamma_{n} y}+c_{n} e^{\Gamma_{n} y}\right) e^{i\left(k_{n}+k_{z}\right) z} .
$$

Here, $d_{n}$ and $c_{n}$ are the dimensionless amplitudes of the counterpropagating waves inside the vacuum gap of the dual-grating structure. $\Gamma_{n}$ is a complex number which describes the transverse variation of the wave. We also introduce a scaling constant $k_{0} / \Gamma_{n}$ as a multiplicative prefactor, which is dimensionless and serves only to simplify the form of the Lorentz force in later discussion.

We also define the parameter $r_{n}$ as the complex ratio of the counterpropagating evanescent field amplitudes when the structure is single-side driven

$$
r_{n}=c_{n} / d_{n}=\left\|r_{n}\right\| e^{i \phi_{r_{n}}}
$$

For a physical interpretation, consider the $+y$ plane wave (top wave, Fig. 1) as it hits the dual grating. It first scatters off the $-y$ (top) grating into the modes supported by the grating. Some fraction of the incident wave remains undiffracted (the zeroth diffraction order) and hits the $+y$ (bottom) grating, which also scatters into the grating modes. If the first grating row is highly reflective, the zeroth order transmission through the top grating is small, the scattered amplitude from the $+y$ (bottom) grating is small while the scattered amplitude from the $-y$ (top) grating remains large, and thus $r_{n}$ is small. In other words, the amplitude of $r_{n}$ is usually controlled by the reflectivity of the single grating row on the side of the incident laser. The two partially reflective grating rows can also cause Fabry-Perot effects, where light reflects back and forth between the two gratings. When Fabry-Perot cavity created by the dual grating is near resonance, $\left|r_{n}\right|$ depends not only on the reflectivity of a single grating but on the characteristics of the Fabry-Perot resonator. Usually, the broadband requirement of DLAs precludes operation near sharp Fabry-Perot resonances. Therefore, in most cases of interest $\left|r_{n}\right|$ is primarily determined by the reflectivity of the first grating row.

The phase of $r_{n}$ is also controllable. It is clearly affected by the width of the channel gap, but also by the position of one grating row relative to the other. "Gliding" one grating along $z$ relative to the other can change the phase of $r_{n}$ (See Sec. VI and Fig. 4). The phase of $r_{n}$ can also be altered by the angle of the illuminating plane wave (See Supplemental Material [18]).
Due to the orthogonality of the spatial harmonics defined by Eq. (3), the modal components of the scattered field must individually obey Eq. (1), which requires each $\Gamma_{n}$ to satisfy a dispersion relation

$$
\left(k_{n}+k_{z}\right)^{2}-\Gamma_{n}^{2}=k_{0}^{2} .
$$

Since our initial focus is on plane wave illumination at normal incidence, the Bloch wave vector $k_{z}=0$. The nonnormal incidence case $\left(k_{z} \neq 0\right)$ is discussed in the Supplemental Material [18].

\section{B. Dual side illumination}

Next we consider excitation of the dual grating by two equal amplitude, normally incident, counterpropagating plane waves, along the $+y$ and $-y$ directions, with a relative phase of $\theta_{r}$ between the two as measured from the center of the channel gap.

We now restrict the discussion to mirror-symmetric dual gratings (Fig. 1). The mirror symmetry of the structure simplifies the problem so that we can discuss the contributions from the dual-grating parameters and the dual-drive phase separately. Both Sec. VI and the Supplemental Material [18] discuss the general, nonsymmetric case in detail.

The mirror symmetry allows us to write the dual-drive fields as

$H_{x}^{(n)}=\frac{E_{0} k_{0}}{\mu_{0} c \Gamma_{n}} e^{i k_{n} z}\left(\left(c_{n}-d_{n} e^{i \theta_{r}}\right) e^{\Gamma_{n} y}+\left(d_{n}-c_{n} e^{i \theta_{r}}\right) e^{-\Gamma_{n} y}\right)$,

where the minus sign is introduced by the action of a mirror transformation on the pseudovector $\mathbf{H}$.

We then rewrite Eq. (9) in terms of hyperbolic functions, and define the amplitude

$$
\epsilon_{n}=2 E_{0} d_{n},
$$

which is equivalent to the dual-drive electric field Fourier coefficient $e_{n}$ used in $[4,12]$ in the limit $\left\|r_{n}\right\| \rightarrow 0$. We note that when $\left\|r_{n}\right\| \neq 0$, the $e_{n}$ coefficient used in previous publications is not exactly equivalent to $\epsilon_{n}$, though both coefficients play the role of an amplitude and global phase scaling coefficient in all cases.

$$
\begin{aligned}
H_{x}^{(n)}= & -\frac{\epsilon_{n}}{\mu_{0} c} \frac{k_{0}}{\Gamma_{n}} e^{i k_{n} z}\left(\left(1+r_{n}\right) \frac{e^{i \theta_{r}}-1}{2} \cosh \left(\Gamma_{n} y\right)\right. \\
& \left.+\left(1-r_{n}\right) \frac{e^{i \theta_{r}}+1}{2} \sinh \left(\Gamma_{n} y\right)\right),
\end{aligned}
$$

where $r_{n}$ is defined in Eq. (7). 
To compress the notation of Eq. (11), we define

$$
a_{n}^{ \pm}=\left(1 \mp r_{n}\right) \frac{e^{i \theta_{r}} \pm 1}{2},
$$

such that

$H_{x}^{(n)}=-\frac{\epsilon_{n}}{\mu_{0} c} \frac{k_{0}}{\Gamma_{n}}\left(a_{n}^{-} \cosh \left(\Gamma_{n} y\right)+a_{n}^{+} \sinh \left(\Gamma_{n} y\right)\right) e^{i k_{n} z}$.

The electric field components corresponding to (13) can be found from Maxwell's equations.

$E_{y}^{(n)}=\epsilon_{n} \frac{k_{n}}{\Gamma_{n}}\left(a_{n}^{-} \cosh \left(\Gamma_{n} y\right)+a_{n}^{+} \sinh \left(\Gamma_{n} y\right)\right) e^{i k_{n} z}$

$E_{z}^{(n)}=i \epsilon_{n}\left(a_{n}^{+} \cosh \left(\Gamma_{n} y\right)+a_{n}^{-} \sinh \left(\Gamma_{n} y\right)\right) e^{i k_{n} z}$.

The Lorentz force acting on an electron traveling with velocity $\beta c \hat{\mathbf{z}}$ is $\mathbf{F}=\Re\left\{[\mathbf{E}(\mathbf{r})+\beta c \hat{\mathbf{z}} \times \mathbf{B}(\mathbf{r})] e^{-i \omega t}\right\}$. To simplify the notation in the following discussion, we focus on the complex form $\tilde{\mathbf{F}}$, which is connected to the force through $\mathbf{F}=\Re\{\tilde{\mathbf{F}}\}$. Re-inserting the harmonic timedependence of the fields to Eqs. (13) and (14), we can now calculate the $n$th harmonic of the Lorentz force $\tilde{\mathbf{F}}_{n}$.

$\tilde{\mathbf{F}}_{n}=q \epsilon_{n} e^{i k_{n} z-i k_{0} c t}\left[\begin{array}{c}0 \\ \xi_{n}\left(a_{n}^{-} \cosh \left(\Gamma_{n} y\right)+a_{n}^{+} \sinh \left(\Gamma_{n} y\right)\right) \\ i\left(a_{n}^{+} \cosh \left(\Gamma_{n} y\right)+a_{n}^{-} \sinh \left(\Gamma_{n} y\right)\right)\end{array}\right]$,

where $q$ is the elementary charge, $\xi_{n}=\left(k_{n}-k_{0} \beta\right) / \Gamma_{n}$, and the vacuum wave number $k_{0}=\omega / c$. The total (complex) Lorentz force is $\tilde{\mathbf{F}}=\sum_{n} \tilde{\mathbf{F}}_{n}$.

To obtain the average Lorentz force in the rigid beam approximation, we assume an electron trajectory described by $z=\beta c t+z_{0}$ over some interaction length $L$, and average $\tilde{\mathbf{F}}_{n}$ to obtain

$$
\begin{aligned}
\overline{\mathbf{F}}_{n}= & q \epsilon_{n} e^{i \frac{k_{0} z_{0}}{\beta}}\left[\begin{array}{c}
0 \\
\xi_{n}\left(a_{n}^{-} \cosh \left(\Gamma_{n} y\right)+a_{n}^{+} \sinh \left(\Gamma_{n} y\right)\right) \\
i\left(a_{n}^{+} \cosh \left(\Gamma_{n} y\right)+a_{n}^{-} \sinh \left(\Gamma_{n} y\right)\right)
\end{array}\right] \\
& \times \frac{1}{L} \int_{-\frac{L}{2}}^{\frac{L}{2}} e^{i\left(k_{n}-\frac{k_{0}}{\beta}\right) z} d z .
\end{aligned}
$$

Since taking the average and taking the real part of $\tilde{\mathbf{F}}_{n}$ are interchangeable, the average Lorentz force of the $n$th harmonic over a length $L$ in the rigid beam approximation is simply $\Re\left\{\overline{\mathbf{F}}_{n}\right\}$.

\section{Phase matching}

Consider now an electron beam of velocity $\beta c$ phasematched to the $n$th spatial harmonic over a single grating period. Over a single period, $\beta$ is very nearly constant, and so the phase of the $n$th harmonic field must be constant with respect to the electron trajectory described by $z=\beta c t+z_{0}$. This yields the phase matching condition

$$
\beta\left(k_{n}+k_{z}\right)=k_{0} .
$$

Over multiple periods, the phase matching condition must be appropriately tapered to retain phase synchronicity [7]. Since we have specialized the discussion to plane wave illumination at normal incidence, $k_{z}=0$, and by Eq. (8), a phase-matched field must satisfy

$$
\Gamma_{n}=k_{n} \sqrt{1-\beta^{2}}=k_{n} / \gamma=k_{0} / \beta \gamma
$$

where

$$
\gamma=1 / \sqrt{1-\beta^{2}}
$$

Thus, all phase-matched modes have $\Re\left\{\Gamma_{n}\right\}>0$, which implies that they are evanescent.

When Eq. (17) is satisfied for the $n$th harmonic, the integral term in Eq. (16) is 1 for the phase-matched harmonic and approximately zero for all other harmonics as long as $L \gg \Lambda$. This means that, in the usual case of $n=1$, the average force on an electron in a dual-grating structure is well approximated by just $\overline{\mathbf{F}}_{1}$.

\section{Lorentz force on phase-matched particle}

The form of the Lorentz force on a phase-matched particle simplifies considerably. For an electron which propagates along $z=\beta c t+z_{0}$, we choose a phasematched electron speed such that $\beta=k_{0} / k_{n}$. The particle is effectively stationary relative to the synchronized wave.

We then explicitly separate the amplitude and phase of $\epsilon_{n}$, defined in Eq. (10), as $\epsilon_{n}=\left\|\epsilon_{n}\right\| e^{i \phi_{\epsilon_{n}}}$. This allows us to define the "electron phase" $\alpha_{n}=k_{0} z_{0} / \beta+\phi_{\epsilon_{n}}$, which is the phase of a reference particle relative to the $n$th phasematched Fourier component of the laser field.

Further, the coefficient $\xi_{n}$ in Eq. (15) simplifies to $1 / \gamma$, where $\gamma$ is defined in Eq. (19), and the average complex Lorentz force is

$\overline{\mathbf{F}}_{n}=q\left\|\epsilon_{n}\right\| e^{i \alpha_{n}}\left[\begin{array}{c}0 \\ \frac{1}{\gamma}\left(a_{n}^{-} \cosh \left(\Gamma_{n} y\right)+a_{n}^{+} \sinh \left(\Gamma_{n} y\right)\right) \\ i\left(a_{n}^{+} \cosh \left(\Gamma_{n} y\right)+a_{n}^{-} \sinh \left(\Gamma_{n} y\right)\right)\end{array}\right]$.

Under symmetric illumination $\left(\theta_{r}=0\right), a_{n}^{-}=0$ and the longitudinal force has a cosh profile along $y$, and is referred to as the "cosh" mode. Under antisymmetric illumination $\left(\theta_{r}=\pi\right), a_{n}^{+}=0$ and the longitudinal force has a sinh distribution-the sinh mode. The sinh mode has a nearly uniform force in the $y$ direction, and is primarily used as a 
beam deflector. Thus, $a_{n}^{+}$and $a_{n}^{-}$are the complex amplitudes of the cosh and sinh modes, respectively. The relative amplitudes of the transverse and longitudinal momentum modulation is determined by the ratio $a_{n}^{-} / a_{n}^{+}$, as well as the electron speed and phase.

The modifications to this equation in the nonphasematched case are found in the Supplemental Material [18].

\section{E. The real Lorentz force in the TM mode}

To obtain the momentum modulation on the interacting electrons, it is useful to have an explicit form of the average real Lorentz force. From Eq. (20),

$\mathbf{F}_{n}=q\left\|\epsilon_{n}\right\|\left[\begin{array}{c}0 \\ \frac{1}{\gamma}\left[\rho_{n}^{-} \cos \frac{\theta_{r}}{2} \sinh \left(\Gamma_{n} y\right)-\sigma_{n}^{+} \sin \frac{\theta_{r}}{2} \cosh \left(\Gamma_{n} y\right)\right] \\ -\left[\rho_{n}^{+} \sin \frac{\theta_{r}}{2} \sinh \left(\Gamma_{n} y\right)+\sigma_{n}^{-} \cos \frac{\theta_{r}}{2} \cosh \left(\Gamma_{n} y\right)\right]\end{array}\right]$,

is the real Lorentz force, where we have defined the real analogues of the complex $a_{n}^{ \pm}$coefficients:

$$
\begin{aligned}
& \rho_{n}^{ \pm}=\left\|1 \pm r_{n}\right\| \cos \left[\alpha_{n}+\frac{\theta_{r}}{2}+\angle\left(1 \pm r_{n}\right)\right], \\
& \sigma_{n}^{ \pm}=\left\|1 \pm r_{n}\right\| \sin \left[\alpha_{n}+\frac{\theta_{r}}{2}+\angle\left(1 \pm r_{n}\right)\right] .
\end{aligned}
$$

$r_{n}$ is defined in Eq. (7). $\angle\left(1 \pm r_{n}\right)$ indicates the phase angle of the quantity $\left(1 \pm r_{n}\right)$ in the complex plane.

Comparing Eq. (21) with previous literature $[2,4,5,10$ 13], it is evident that the dependence of the Lorentz force on the electron phase $\alpha_{n}$, the dual-drive phase $\theta_{r}$, and the "reflection phase" $\phi_{r_{n}}$ is more complex than previously reported. However, these equations exactly match the literature in the common limit of high-reflectivity gratings $\left(r_{n} \rightarrow 0\right)$ operated on pure cosh and sinh modes $\left(\theta_{r}=0\right.$ or $\pi$ ).

\section{SYMMETRIES OF THE TM-MODE FORCES}

The Lorentz force in the mirror-symmetric dual gratings shown in Eqs. (20) and (21) manifests different symmetries under different conditions. The various symmetries provide a powerful tool for understanding the tuning of dual-grating devices and the correspondence between single-side and dual-drive illumination.

We can rewrite Eq. (20) in the following form:

$$
\left[\begin{array}{c}
\bar{F}_{n, z} \\
\bar{F}_{n, y}
\end{array}\right]=i q\left\|\epsilon_{n}\right\| e^{i \alpha_{n}}\left(1-r_{n}^{2}\right)^{\frac{1}{2}}\left[\begin{array}{cc}
1 & 0 \\
0 & \frac{1}{\gamma}
\end{array}\right] U\left[\begin{array}{c}
\cosh \left(\Gamma_{n} y\right) \\
\sinh \left(\Gamma_{n} y\right)
\end{array}\right],
$$

where

$$
U=e^{i \frac{\theta_{r}}{2}}\left[\begin{array}{cc}
\zeta \cos \frac{\theta_{r}}{2} & -\zeta^{-1} \sin \frac{\theta_{r}}{2} \\
\zeta^{-1} \sin \frac{\theta_{r}}{2} & \zeta \cos \frac{\theta_{r}}{2}
\end{array}\right]\left[\begin{array}{cc}
1 & 0 \\
0 & -i
\end{array}\right],
$$

and

$$
\zeta=\left(\frac{1-r_{n}}{1+r_{n}}\right)^{\frac{1}{2}}
$$

The longitudinal and transverse forces can be expressed as a transformation $U$ acting on a basic vector $\mathbf{v}=$ $\left[\cosh \left(\Gamma_{n} y\right), \sinh \left(\Gamma_{n} y\right)\right]$. By tuning the relative phase $\theta_{r}$ and designing a DLA structure with a specific $r_{n}$, we control the operator $U$ acting on $\mathbf{v}$ and thus control the force on the synchronized electrons.

\section{A. High reflectivity limit}

In the limit of highly reflective gratings $\left(\left\|r_{n}\right\| \rightarrow 0\right), \zeta \rightarrow 1$ and $U$ becomes an element of the Lie group $\mathrm{U}(2)$. By tuning $\theta_{r}$, the amplitude of the cosh (and sinh) components can be varied from maximum to zero (zero to maximum). In this limit, the cosh and sinh modes have the same maximal amplitude, but are $\pi / 2$ out-of-phase. Previous dual-drive experiments tend to favor this limit, due to its operational simplicity $[2,3]$.

\section{B. High transmissivity limit}

In the highly transmissive limit $\left(\left\|r_{n}\right\| \rightarrow 1\right), \quad \zeta=$ $\sqrt{-i}\left(\tan \frac{\phi_{r_{n}}}{2}\right)^{\frac{1}{2}}$ and the operator $U$ becomes

$$
U=\sqrt{-i} e^{i \theta_{r}}\left[\begin{array}{cc}
\left(\tan \frac{\phi_{r_{n}}}{2}\right)^{\frac{1}{2}} \cos \frac{\theta_{r}}{2} & -\left(\cot \frac{\phi_{r_{n}}}{2}\right)^{\frac{1}{2}} \sin \frac{\theta_{r}}{2} \\
i\left(\cot \frac{\phi_{r_{n}}}{2}\right)^{\frac{1}{2}} \sin \frac{\theta_{r}}{2} & -i\left(\tan \frac{\phi_{r_{n}}}{2}\right)^{\frac{1}{2}} \cos \frac{\theta_{r}}{2}
\end{array}\right] .
$$

In contrast to the highly reflective gratings, the operator $U$ is no longer unitary. Though tuning the relative phase $\theta_{r}$ can still change the relative amplitudes of the cosh and sinh modes, the maximal amplitudes of the cosh and sinh modes are influenced by the phase of $r_{n}$. When $\phi_{r_{n}} \approx(2 m+1) \pi$, $m \in \mathbb{Z}$, the cosh mode is dominant, while the sinh mode dominates when $\phi_{r_{n}} \approx 2 m \pi$, regardless of $\theta_{r}$. Further, the amplitudes of the cosh and sinh modes are either in-phase or $\pi$ out-of-phase. Such properties are also found in multichannel DLAs where, by necessity, each grating row has high transmissivity [26].

A further symmetry manifests when $\left\|r_{n}\right\| \rightarrow 1$ and $r_{n}$ can be written as a pure phase term $r_{n}=e^{i \phi_{r_{n}}}$. Equation (12) becomes

$$
a_{n}^{ \pm}=\frac{\left(1 \mp e^{i \phi_{r_{n}}}\right)\left(e^{i \theta_{r}} \pm 1\right)}{2} .
$$


The interchange of $\phi_{r_{n}}$ and $\theta_{r}$ causes $a_{n}^{ \pm} \leftrightarrow-a_{n}^{\mp}$. The form of Eq. (20) is preserved, with the cosh and sinh terms exchanging roles and flipping sign. This implies that in the highly transmissive limit $\phi_{r_{n}}$ plays an almost identical role to the dual-drive phase $\theta_{r}$, and by tuning it, one can replicate nearly all the properties of a dual-drive structure.

\section{CLASSIFICATION OF MODES BY PHASE-SPACE PROFILES}

The transverse and longitudinal momentum kicks felt by a phase-matched electron over one grating period follow directly from integration of Eq. (21) over $z$. For a phasematched particle, Eq. (21) has constant phase and thus no $z$-dependence, and so the momentum kicks over one period $\Lambda$ are given simply by multiplication of the Lorentz force by the time-of-flight for one grating period: $\Delta \mathbf{p}_{n}=\frac{\Lambda}{\beta c} \mathbf{F}_{n}$. The mismatched case is only slightly different. It has a $z$-dependent phase, and so an integral must be carried out explicitly.

It is instructive to make plots of the phase space furnished by $\left(\Delta p_{\perp}, \Delta p_{\|}\right)$as the electron phase $\alpha_{n}$ varies over the interval $[0,2 \pi)$, because the patterns drawn in this space correspond exactly to the experimentally measured electron beam profiles seen in [2,5] and many other studies. Parallel and perpendicular kicks are defined relative to the electron reference trajectory along the $z$-axis.

Consider the phase space profiles produced by an electron beam traveling on-axis $(y=0)$. There are three distinct profiles that can be produced in $\left(\Delta p_{\perp}, \Delta p_{\|}\right)$space. The linelike profiles which rotate around a central point as $\theta_{r}$ is varied, upright ellipses whose major and minor axes grow and shrink with $\theta_{r}$, and elliptical profiles which both rotate and stretch with $\theta_{r}$ (See Fig. 2).

The linelike profile observed by Leedle et al. [2] occurs when $\angle\left(1+r_{n}\right)=\angle\left(1-r_{n}\right)$, which is satisfied only when $r_{n} \in \mathbb{R}$. Variation of the dual-drive phase $\theta_{r}$ manifests as clockwise rotation of the line by angle $\varphi$ relative to the $\Delta p_{\|}$ axis (See Supplemental Material [18], Rotation Angle of Modal Profiles section, for an implicit form of $\varphi$ ).

The upright ellipse is produced when $\left\|r_{n}\right\|=1$. If $r_{n}$ is also real (i.e., $r_{n}= \pm 1$ ), then this ellipse has zero width, and manifests as a line on the $\Delta p_{\perp}$ or $\Delta p_{\|}$axis which grows and shrinks with $\theta_{r}$.

The most general case occurs when none of the previous conditions are satisfied, i.e., $r_{n} \in \mathbb{C}$-the profile is a general rotated ellipse. The rotation angle $\varphi$ depends on both the dual drive phase $\theta_{r}$ and the ratio $r_{n}$. An implicit form on $\varphi$ is given in the Supplemental Material [18].

Through the Lorentz force, we can establish a mapping from the dual-grating and illumination characteristics $\left(r_{n}\right.$, $\theta_{r}$, etc.) to the momentum modulation of the phasematched electrons, and thus to the mode profiles observed in experiment. Equipped with this mapping, one can design the dual grating and tune the experimental configuration to

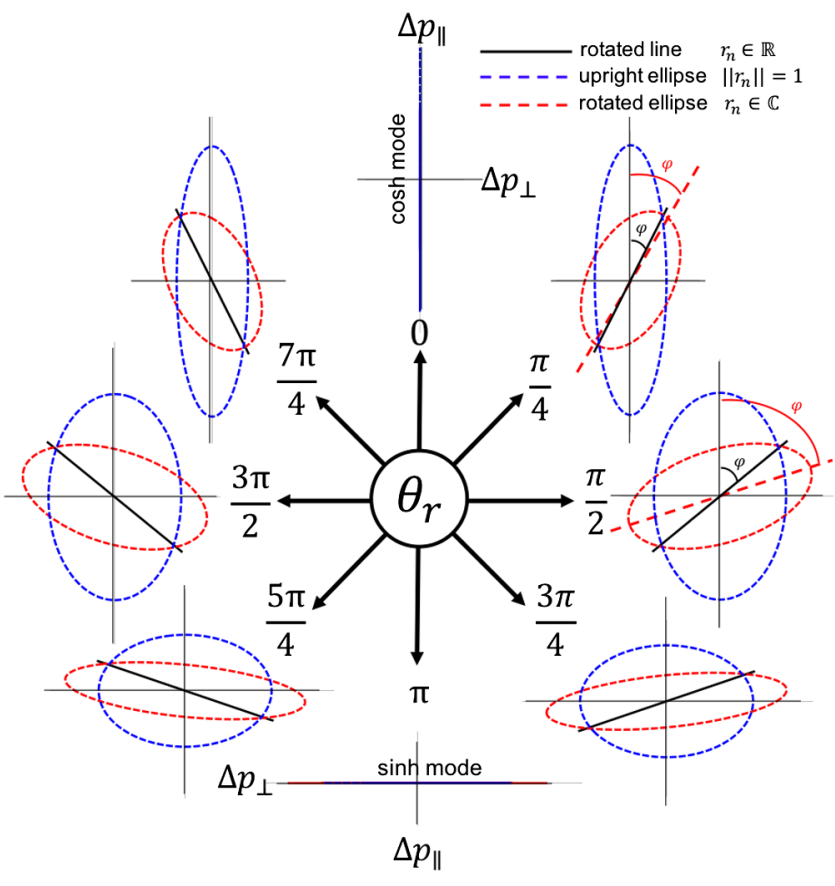

FIG. 2. The $\left(\Delta p_{\perp}, \Delta p_{\|}\right)$phase space is plotted for the three classes of modes. The (normalized) momentum kicks plotted here are derived by integration of the $n=1$ component of Eq. (21) for an on-axis particle $(y=0), \gamma$ is set to 1 for convenience. As $\alpha$ varies from 0 to $2 \pi$, it traces out ellipses in $\left(\Delta p_{\perp}, \Delta p_{\|}\right)$space. Values of $r_{n}$ corresponding to the three mode classes are then chosen, and $\theta_{r}$ is varied from 0 to $2 \pi$ for each mode. The rotated line (solid black) has $r_{1}=0.1$. The upright ellipse (dashed blue) has $r_{1}=e^{2 i}$. The general ellipse (dashed red) has $r_{1}=0.7 e^{i}$. The principal modes occur when $\theta_{r}=0$ or $\pi$. All other $\theta_{r}$ values produce skew modes.

achieve the desired operational mode. In addition, by observation of the electron beam profiles under different dual drive phases, this mapping can provide valuable information about the dual-grating characteristics.

Via tuning of $\theta_{r}$ and $r_{n}$, the whole phase-space of dualgrating operation modes can be explored. Previous studies, such as those presented in $[2,4,11]$, predict the upright elliptical profile in $\left(\Delta p_{\perp}, \Delta p_{\|}\right)$space, which agrees with experiment only when $\theta_{r}=0$ or $\pi$. However, the previous equations cannot explain the rotated line modes observed by Leedle et al. for $\theta_{r} \neq 0$ or $\pi$ in [2], which are rotated by some angle corresponding to the dual-drive phase. The equations presented here correctly reproduce the line modes if $r_{n}$ is real. Further, they capture all operating modes of the dual-grating structures thus far observed and predict a new mode, the rotated ellipse. The correspondence between $\theta_{r}$ and $\phi_{r_{n}}$ also suggests that these modes should be accessible to both single and dual-side drive operation of dual-grating structures.

In practical design of dual pillar DLAs, the desired $r_{n}$ can be achieved through parameter tuning, and a simple concrete geometrical design rule may not be necessary. 
For simple geometries like ellipses and rectangles, even brute-force parameter sweeps can provide a design with desired $r_{n}$. In some special designs like the multichannel photonic crystal DLAs, the value of $r_{n}$ is associated with the band structure properties of the underlying photonic crystal, which is discussed further in [26].

\section{SINGLE-SIDE DRIVE}

Although the full phase-space of operation modes discussed in Sec. IV can be achieved using dual-side illumination, it is experimentally simpler in some cases to use only single-side illumination. Both the field and force equations for single-side illumination can be recovered immediately from Eq. (20) by setting $e^{i \theta_{r}}$ to 0 in the definition of $a_{n}^{ \pm}$. With single-side illumination, $r_{n}$ depends primarily on the dual-grating design, though angled illumination will also affect $r_{n}$ (See Supplemental Material [18]). By tuning the geometries of the two gratings, $r_{n}$ can, in principle, be any complex number. Therefore, the whole space of modes, as illustrated in Fig. 2 can be explored with single-side illuminated dual gratings.

In the limit $r_{n} \rightarrow 1$ (a perfectly transmissive grating), the single-illumination gratings behave very similarly to dualilluminated gratings, where the phase of the $r_{n}$ term plays the role of $\theta_{r}$. Up to a sign, $\phi_{r_{n}}$ enters into Eq. (20) in exactly the same fashion as $\theta_{r}$. Thus, the effects of $\phi_{r_{n}}$ rotation on a singly illuminated DLA should precisely match that of $\theta_{r}$ rotation on a dual-illuminated DLA. The converse is also true- the tunability provided by dualillumination can be used to partially compensate for fabrication error in $r_{n}$, or dynamically alter the properties and mode of a DLA device with fixed $r_{n}$.

Figure 3 shows the elliptical profile produced by a single-sided illumination of a dual-pillar DLA structure with a design similar to [2] and [5]. The elliptical mode in Fig. 3 appears slightly skewed, and thus corresponds to the general elliptical case where $r_{n} \in \mathbb{C}$ (the red line shown in Fig. 2). This is the first time that an elliptical mode profile has been observed. The dual-pillar structure used to produce this mode was originally designed as a highefficiency cosh mode structure, but produces the elliptical profile seen in Fig. 3 when illuminated at a slight angle. Angled illumination of dual-grating structures changes the effective $r_{n}$ coefficient, and thus provides a good example of the use of this $r_{n}$ parameter to design the phase space modulation of an electron beam. A more powerful method of tuning $r_{n}$ is explored in Sec. VI, and angle tuning is treated in more detail in the Supplemental Material [18].

Further experimentation that fully explores the phase space profiles predicted by Eq. (21) requires dedicated fabrication of devices designed for production of elliptical modes, as well as improvement of the electron beam pointspread function so that the profiles are easier to distinguish. Based on the theory presented here, it should be possible to

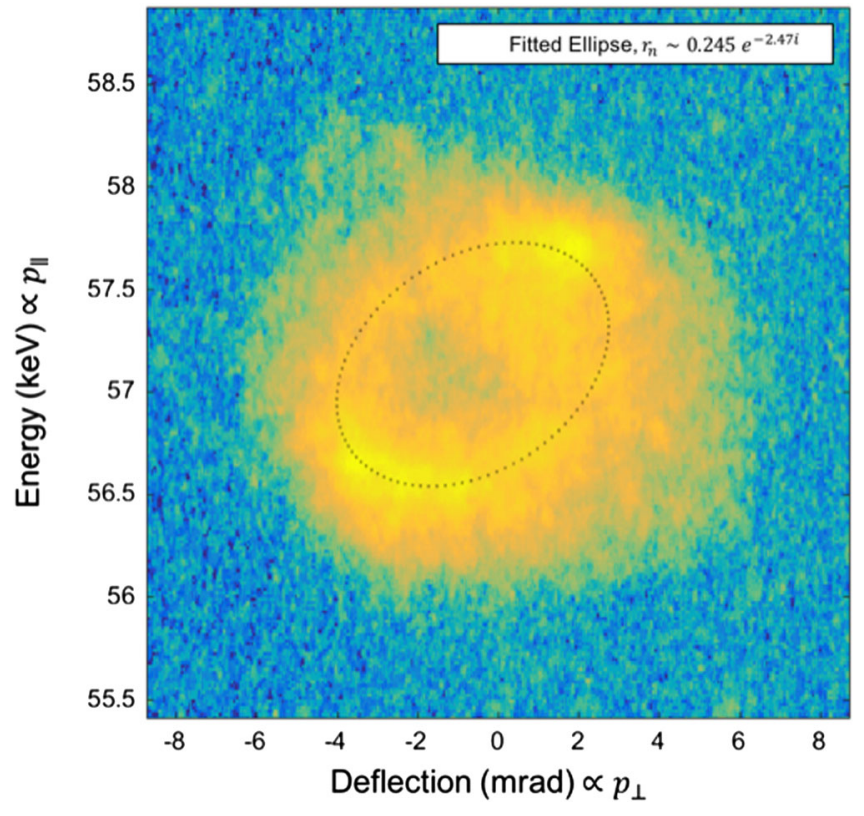

FIG. 3. This figure shows an elliptical profile produced by single-side illumination of a dual-pillar DLA device. The structure was originally designed for cosh mode operation, but can be made to produce elliptical beam profiles through suitable angle tuning. For a $57 \mathrm{keV}$ beam, an energy gain of $500 \mathrm{eV}$ corresponds to a longitudinal momentum kick of roughly $1.1 \mathrm{keV} / \mathrm{c}$, and a $5 \mathrm{mrad}$ deflection corresponds to a transverse momentum kick of roughly $1.2 \mathrm{keV} / \mathrm{c}$. Note: The $r_{n}$ value for this ellipse was extracted by an elliptical fitting algorithm in MATLAB. The fit error is unknown due to large uncertainty in the horizontal (deflection) axis calibration. Please see the Supplemental Material [18] for experimental details.

probe the space of all possible modes with either single or dual-side drive structures.

An elliptical mode, or better yet perfectly circular mode, has a potential use case in attosecond-scale temporal streaking measurements. Attosecond-scale measurements of electron bunch length have previously been accomplished by electron beam streaking either in the energy dimension or in angle [5,8,27-31]. However, because the resolution of any single-axis streaking measurement is proportional to the instantaneous slope of the streaking field, the resolution of these measurements is identically zero at the field extrema. Consider a skew mode like the black line in Fig 2 . The temporal resolution is identically zero at the edges of the mode, corresponding to $\alpha=0$ and $\pi$. The resolution is only maximized at the field zerocrossing (the center of the line profile). Additionally, due to the linear profile, the intervals $\alpha=[0, \pi)$ and $\alpha=[\pi, 2 \pi)$ physically overlap, reducing the "analysis window" to one half the optical cycle.

However, a streaking measurement that traces a circle in the $2 \mathrm{D}$ phase space furnished by $\left(p_{\perp}, p_{\|}\right)$can have maximal resolution at all phases. In a circular mode, there are no extrema that occur simultaneously in both the 
$p_{\perp}$ and $p_{\|}$dimension. Additionally, the circular mode only overlaps itself once per optical cycle, automatically doubling the analysis window. This streaking method requires a $2 \mathrm{D}$ electron detector.

\section{GLIDING OF DUAL GRATINGS}

Thus far, we have considered only dual-gratings that have mirror symmetry. When this restriction is relaxed, we find that gliding one grating row along $z$ with respect to the other provides a powerful degree of freedom in the design of dual-grating DLAs, which is especially critical for single-side drive structures [16]. In particular, the phase $\phi_{r_{n}}$ can be tuned independently of the amplitude $\left\|r_{n}\right\|$ by gliding one grating row with respect to the other.

When the near-field coupling of the two gratings is weak, a gliding operation has negligible influence on the amplitude of $r_{n}$, which is determined by the transmissivity of the first row of grating and the diffraction strength of the two gratings. The near-field coupling, where the channel width is $w$, is characterized by $\exp \left(-\Gamma_{n} w\right)$, which is generally small for subrelativistic DLAs.

To demonstrate the tuning of the phase of $r_{n}$ through gliding, we numerically investigate a nonsymmetric dual grating under single-side drive. We define the glide ratio $g=\delta l / \Lambda$ as the ratio between the translation $\delta l$ of one row of grating along $z$-direction and the periodicity of the grating $\Lambda$, as illustrated in Fig. 4(a).

Since the specific pillar geometry is not important to demonstrate the effect of gliding, we choose simple rectangular silicon pillars to form the dual gratings. To phase match electrons with $\beta=0.5$ with an illuminating wavelength $\lambda$ of $2 \mu \mathrm{m}$, we choose a grating period $\Lambda$ of $1 \mu \mathrm{m}$ (and use the $n=1$ diffraction order). We also choose the rectangle's parameters such that transmissivity of a single grating row is above $90 \%$. The rectangular pillars have width and length of $0.52 \mu \mathrm{m}$ and $1.32 \mu \mathrm{m}$, respectively. The electron channel width $w$ is $0.4 \mu \mathrm{m}$. The field distribution for $g=0.5$ is shown in Fig. 4(b).

As the glide ratio increases from 0 to 1 , the amplitude of $r_{n}$ is roughly constant, as shown in Fig. 4(c) (See Supplemental Material [18] for fitting $r_{n}$ from numerical simulations). However, the phase of $r_{n}$ changes almost linearly with the glide ratio [Fig. 4(c)]. This modulates the relative amplitudes of the cosh and sinh modes, as shown in Fig. 4(d). The gradual tuning of the longitudinal force profile here is very similar to the dual drive phase modulation. For a single-side illumination structure, tuning the glide ratio of a high-transmissivity dual grating provides the same functionality as tuning the dual-drive phase in a dual-drive configuration. When $\left\|r_{n}\right\| \approx 1$, pure cosh or sinh modes can be achieved. This provides a systematic approach to achieve arbitrary operation modes in the phase-space even with single-side illumination.

When the symmetric dual gratings are offset by exactly $1 / 2$ period, forming "glide-symmetric" gratings, it is
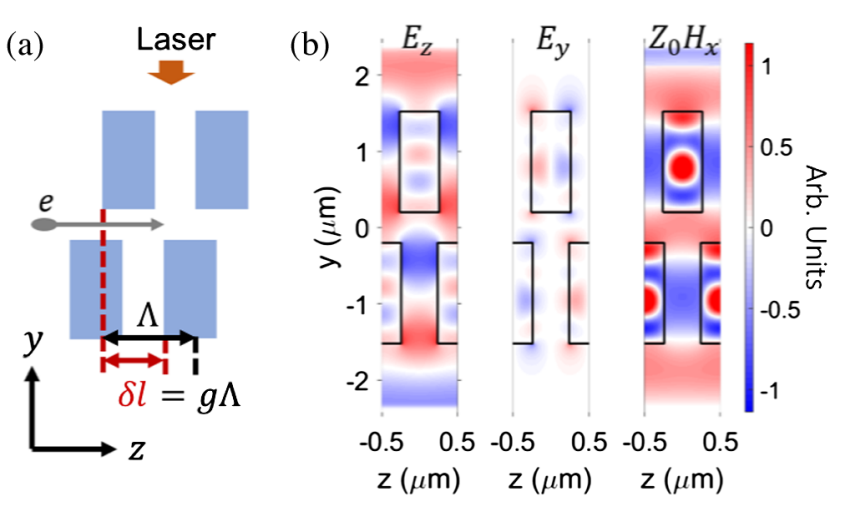

(c)

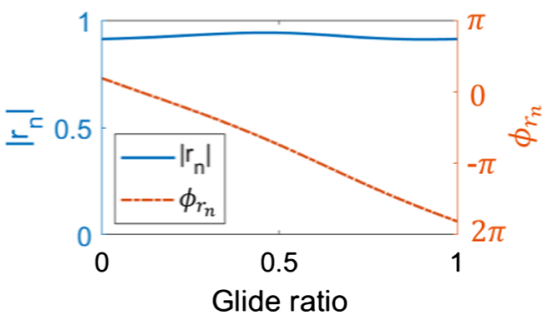

(d)

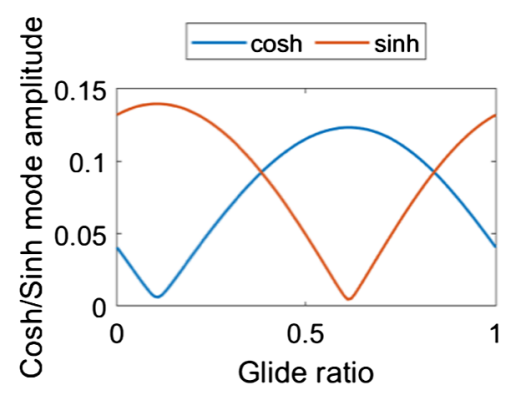

FIG. 4. (a) Gliding one row of grating with respect to the other. The dual grating consists of two rows of rectangular silicon pillars with width $0.52 \mu \mathrm{m}$, length $1.32 \mu \mathrm{m}$, periodicity $1 \mu \mathrm{m}$ and electron channel width $0.4 \mu \mathrm{m}$. The illumination laser wavelength is $2 \mu \mathrm{m}$ and electron velocity is $0.5 c$. (b) shows the electric and magnetic field of the dual grating with glide ratio $g=0.5$ and top-side illumination. The amplitude and phase of $r_{n}$ through tuning the glide ratio are shown in (c). (d) shows the amplitudes of the cosh and sinh modes as a function of the glide ratio.

possible to excite pure cosh or sinh modes in a very similar manner to mirror-symmetric gratings. An analytic description of these gratings and their special properties is given in the Supplemental Material [18].

\section{CONCLUSION}

In this manuscript, we generalize previous studies to dual-grating DLAs with arbitrary illumination conditions. In doing so, we fully classify the modes producible by single DLA structures in both single-side drive and dualdrive configurations. We provide a framework that unifies the description of linelike skew modes previously observed by Leedle et al. in [2] with the previously predicted upright elliptical modes, and both predict and experimentally demonstrate a novel rotated-ellipse mode profile. 
Additionally, we highlight the flexibility of the structures currently employed in DLA research - they are capable of arranging electron beams into diverse phase space profiles with many potential use cases. As an example, while a pure accelerating structure requires a linear profile, a circular profile could have superior resolution in a streaking measurement.

Further, the symmetries of the Lorentz force equations imply that the tunability of a dual-drive system can be entirely replicated in a single-side drive system by suitable tuning of the structure geometry and/or illumination conditions (angle, etc.). Through symmetry arguments and discussion of the glide ratio, we offer complementary methods of designing the electron phase space for both single and dual-side drive configurations.

It is our hope that this study will assist in the future development of accelerators and other sophisticated beam manipulation devices based on the DLA architecture.

\section{ACKNOWLEDGMENTS}

This work is funded by the Gordon and Betty Moore Foundation (GBMF4744).

[1] D. Cesar, S. Custodio, J. Maxson, P. Musumeci, X. Shen, E. Threlkeld, R. J. England, A. Hanuka, I. V. Makasyuk, E. A. Peralta, K. P. Wootton, and Z. Wu, High-field nonlinear optical response and phase control in a dielectric laser accelerator, Commun. Phys. 1, 46 (2018).

[2] K. J. Leedle, D. S. Black, Y. Miao, K. E. Urbanek, A. Ceballos, H. Deng, J. S. Harris, O. Solgaard, and R. L. Byer, Phase-dependent laser acceleration of electrons with symmetrically driven silicon dual pillar gratings, Opt. Lett. 43, 2181 (2018).

[3] K. J. Leedle, A. Ceballos, H. Deng, O. Solgaard, R. F. Pease, R. L. Byer, and J. S. Harris, Dielectric laser acceleration of sub-100 keV electrons with silicon dual pillar grating structures, Opt. Lett. 40, 4344 (2015).

[4] D. S. Black, K. J. Leedle, Y. Miao, U. Niedermayer, R. L. Byer, and O. Solgaard, Laser-Driven Electron Lensing in Silicon Microstructures, Phys. Rev. Lett. 122, 104801 (2019).

[5] D. S. Black, U. Niedermayer, Y. Miao, O. Solgaard, R. L. Byer, and K. J. Leedle, Net Acceleration and Direct Measurement of Attosecond Electron Pulses in a Silicon Dielectric Laser Accelerator, Phys. Rev. Lett. 123, 264802 (2019).

[6] J. McNeur, M. Kozák, N. Schönenberger, K. J. Leedle, H. Deng, A. Ceballos, H. Hoogland, A. Ruehl, I. Hartl, R. Holzwarth, O. Solgaard, J. S. Harris, R. L. Byer, and P. Hommelhoff, Elements of a dielectric laser accelerator, Optica 5, 687 (2018).

[7] U. Niedermayer, T. Egenolf, O. Boine-Frankenheim, and P. Hommelhoff, Alternating-Phase Focusing for DielectricLaser Acceleration, Phys. Rev. Lett. 121, 214801 (2018).
[8] N. Schönenberger, A. Mittelbach, P. Yousefi, J. McNeur, U. Niedermayer, and P. Hommelhoff, Generation and Characterization of Attosecond Microbunched Electron Pulse Trains via Dielectric Laser Acceleration, Phys. Rev. Lett. 123, 264803 (2019).

[9] B. Naranjo, A. Valloni, S. Putterman, and J. B. Rosenzweig, Stable Charged-Particle Acceleration and Focusing in a Laser Accelerator Using Spatial Harmonics, Phys. Rev. Lett. 109, 164803 (2012).

[10] T. Plettner, R. L. Byer, C. McGuinness, and P. Hommelhoff, Photonic-based laser driven electron beam deflection and focusing structures, Phys. Rev. Accel. Beams 12, 101302 (2009).

[11] J. Breuer, J. McNeur, and P. Hommelhoff, Dielectric laser acceleration of electrons in the vicinity of single and double grating structures-Theory and simulations, J. Phys. B. 47, 234004 (2014).

[12] U. Niedermayer, T. Egenolf, and O. Boine-Frankenheim, Beam dynamics analysis of dielectric laser acceleration using a fast $6 \mathrm{D}$ tracking scheme, Phys. Rev. Accel. Beams 20, 111302 (2017).

[13] R. J. England, A. Ody, and Z. Huang, Transverse forces in planar symmetric dielectric laser-driven accelerators, Report No. SLAC-PUB-17450, 2019.

[14] Z. Chen, K. Koyama, M. Uesaka, M. Yoshida, and R. Zhang, Resonant enhancement of accelerating gradient with silicon dual-grating structure for dielectric laser acceleration of subrelativistic electrons, Appl. Phys. Lett. 112, 034102 (2018).

[15] L. Pilozzi, A. D'Andrea, and R. Del Sole, Electromagnetic properties of a dielectric grating. I. Propagating, evanescent, and guided waves, Phys. Rev. B 54, 10751 (1996).

[16] D. B. Cesar, Probing ultrafast dynamics with relativistic electrons, Ph.D. thesis, UCLA, 2019.

[17] D. J. Griffiths, Introduction to Electrodynamics, (PrenticeHall, Upper Saddle River, N.J., 1999).

[18] See Supplemental Material at http://link.aps.org/ supplemental/10.1103/PhysRevAccelBeams.23.114001 contains useful extensions of the theory provided in the main text, as well as a discussion on the materials and methods used to generate the experimental data in the main text, which includes Ref. [19-25].

[19] T. Plettner and R. L. Byer, Proposed dielectric-based microstructure laser-driven undulator, Phys. Rev. Accel. Beams 11, 030704 (2008).

[20] S. J. Smith and E. M. Purcell, Visible light from localized surface charges moving across a grating, Phys. Rev. 92, 1069 (1953).

[21] O. A. Tret'yakov, E. I. Chernyakov, and V. P. Shestopalov, Theory of the Smith-Purcell Effect, Sov. Radiophys. 9, 219 (1966).

[22] T. Hughes, G. Veronis, K. P. Wootton, R. J. England, and S. Fan, Method for computationally efficient design of dielectric laser accelerator structures, Opt. Exp. 25, 15414 (2017).

[23] P. Yousefi, N. Schönenberger, J. Mcneur, M. Kozák, U. Niedermayer, and P. Hommelhoff, Dielectric laser electron acceleration in a dual pillar grating with a distributed Bragg reflector, Opt. Lett. 44, 1520 (2019). 
[24] J. Breuer and P. Hommelhoff, Laser-based Acceleration of Nonrelativistic Electrons at a Dielectric Structure, Phys. Rev. Lett. 111, 134803 (2013).

[25] A.C. Ceballos, Silicon photocathodes for dielectric laser accelerators, Ph.D. thesis, Stanford University, 2019.

[26] Z. Zhao, D. S. Black, R. Joel England, T. W. Hughes, Y. Miao, O. Solgaard, R. L. Byer, and S. Fan, Design of a multichannel photonic crystal dielectric laser accelerator, Photonics Res. 8, 1586 (2020).

[27] C. Kealhofer, W. Schneider, D. Ehberger, A. Ryabov, F. Krausz, and P. Baum, All-optical control and metrology of electron pulses, Science 352, 429 (2016).
[28] P. Eckle, M. Smolarski, P. Schlup, J. Biegert, A. Staudte, M. Schöffler, H. G. Muller, R. Dörner, and U. Keller, Attosecond angular, Nat. Phys. 4, 565 (2008).

[29] J. Itatani, F. Quéré, G. L. Yudin, M. Y. Ivanov, F. Krausz, and P. B. Corkum, Attosecond Streak Camera, Phys. Rev. Lett. 88, 173903 (2002).

[30] Y. Morimoto and P. Baum, Diffraction and microscopy with attosecond electron pulse trains, Nat. Phys. 14, 252 (2018).

[31] F. O. Kirchner, A. Gliserin, F. Krausz, and P. Baum, Laser streaking of free electrons at $25 \mathrm{keV}$, Nat. Photonics 8, 52 (2014). 